\title{
Dissemination of Word of Mouth Based on SNA Centrality Modeling and Power of Actors - An Empirical Analysis of Internet Word of Mouth
}

\author{
Feixiang Zhang ${ }^{1} \&$ Liyong Zong $^{2}$ \\ ${ }^{1}$ College of Communication and Art Design, University of Shanghai for Science and Technology, Shanghai, China \\ ${ }^{2}$ Shanghai Publishing and Printing College, Shanghai, China \\ Correspondence: Feixiang Zhang, College of Communication and Art Design, University of Shanghai for Science \\ and Technology, Jungong Road 516, Yangpu District, Shanghai 200093, China. E-mail: Zhangfx2000@sina.com
}

Received: August 21, 2014

Accepted: September 2, 2014

Online Published: September 5, 2014

doi:10.5430/ijba.v5n5p65

URL: http://dx.doi.org/10.5430/ijba.v5n5p65

\begin{abstract}
Combined with social network analysis (SNA), the actors' dissemination net of word of mouth is analyzed, and the network of "concern" and "being concerned" is described. With index of centrality measure, three aspects of the actors in the word-of-mouth network are discussed in terms of the degree centrality, betweenness centrality, closeness centrality. The dimensions of the power of actors are divided, and the sample data of word-of-mouth spreading is analyzed with hierarchical clustering. Finally some conclusions and marketing implications are put forward.
\end{abstract}

Keywords: SNA, centrality, word-of-mouth, power

\section{Introduction}

People's social interaction generally refers to information communication, work negotiation and emotion expression in homogeneous or affinity groups, word of mouth (WOM) dissemination is usually associated with these social activities. Some scholars have studied related issues on word-of-mouth in view of social networks theory. Brown \& Reingen (1987) analysed the spreading path of word-of-mouth, and diagramed a consumer word-of-mouth recommendation social network to study effect of WOM in interpersonal contac (Brown J J, Reingen P H, 1987). Bristor (1990) discussed influence of interpersonal relation on WOM dissemination and pointed that the essence of WOM dissemination formed by people in the social network (Bristor J M., 1990). Tang Jianmin etc. (2008) adopts SNA method to discuss WOM dissemination, some related problem analysed from the network center degrees, network range, network strength and network stability(Tang JianMin, zhu Sai., 2008). Through a customer word-of-mouth dissemination network, Yu Hongyi (2009) analyzed the WOM spreading with some social network parameter, such as network clique, network density, network accessibility and degree centrality (Yu Hongyi, 2009). Li Lin, etc (2009) used complex network dissemination theory to analyse the social network service quality communication problem (Li Lin, Sun Jun-Hua \& Zhou Zhang-Jin, 2009). In addition, also some scholars used the concept of social network to analyze word-of-mouth dissemination of online community (Yang Xue-Cheng \& Zhang Xiao-Hang, 2009).

As we all known, whether based on realistic social face-to-face oral communication, or on virtual community in the Internet, word of mouth is an important way for business marketing. And marketers can spread valuable information to consumer through reality or virtual relation network, and WOM information dissemination path or network structure has significant influence on marketing and communication effect. So the WOM dissemination paths and network study not only catches management practitioners' interest and attention, it's also an important issue discussed by relevant academic researchers.

In SNA, different methods has different research orientation, SNA includes ego-networks analysis and whole network analysis. "Centrality" of ego-networks analysis is the key measure indicator which can show the position of individual in a network (Freeman L C, 1977).

As one important part of ego-networks analysis, "centrality" is used to discuss the degree of central position of individual or organization in the social network. Therefore, the study of centrality of social network in WOM dissemination is of great importance to the analysis of information path in the process of WOM dissemination and its 
dissemination effect.

Now there is less research literature on centrality of social network in WOM dissemination. This paper uses a set of data of micro bloggers to analyze social network of WOM dissemination from the perspective of SNA by adopting the concepts of "centrality" and node "power" types in combination of three measurements of centrality.

\section{The Introduction of Social Network of WOM Dissemination}

Social network is a collection composed by connections between various actors and each actor (the relationship between actors). Both senders of WOM information and its recipients can be viewed as actors in social networks and WOM dissemination can be seen as information transmission relationship among the actors.

In view of the basic properties of social networks of both traditional WOM and IWOM in terms of transmission path and transmission network essence in communication, traditional WOM dissemination and WOM dissemination of virtual community will be explored by adopting the relevant theories.

The study of IWOM communication is the extension of WOM communication theories and can draw on the research on the traditional WOM transmission path. All in all, the studies of WOM dissemination process under the different communication contexts can be instructive to each other, enriching the theoretical study of WOM communication.

In this paper, considering that microblog network has relatively stable state and it easy to observe, a small microblog network is chosen as the research object.

The transmission in microBlogs is based on certain communication models of social networks rather than random and ruleless relations between people.The difference of people's education, interest and professional background leads to their varied preference for information. So people select to concern some users according to their different interests and then a virtual "social network" is formed. The paper adopts the method of "rolling snowball" in sampling, which means over 100 thousand of microblog users he or she "concerns" will be recorded by observing after identifying one microblog user randomly. Accordingly, the same method will be used to observe and record these recorded microblog users.

After observation records, the user "concern" and "concerned" relationship can be described with an adjacency matrix. In this matrix, rows means "concern" relationship, column means "concerned" relationship. "1"on the point of intersection means there is one "concern" and "concerned" relationship existing, and "0" means no such a relationship exists. This matrix can be defined as "concern matrix". In the directed relationship, each node represents a user, and the connections between them represent the relationship of "concern" and "concerned".

The arrow pointing from node to node B means A "concern " $\mathrm{B}$. The number of arrows from a node to other nodes is named as outdegree while the number of arrows of a node being pointed by other nodes is named indegree. Because there exist such directed relationships among these nodes, if A "concerns" B, the news released by B will be shown soon in A's microblog page. That is, B sends the information to A.

\section{The Analysis of Social Network Actors' Centrality in WOM Dissemination}

When "centrality" is used to measure networks in practice, Freeman (1979) pointed out that "the centrality of an entire network should index the tendency of a single pint to be more central than all other points in the network" (Freeman LC, 1979).

Combined with the relationship of "concern" and "concerned" between microblog users, the centrality of different actors in IWOM dissemination can be found by analyzing central degrees and central potential indexes. Input the above mentioned "concern matrix" into UCINET software to measure its degree centrality, betweenness centrality and closeness centrality.

Measurement Indexes of degree centrality UCINET software outputs include: OutDegree, InDegree, NrmOutDeg, NrmInDeg. Measurement Indexes of betweenness centrality include: Betweenness, nBetweenness. Measurement Indexes of closeness centrality include: inFarness, outFarness, inCloseness, outCloseness. To facilitate analysis, the absolute degree centrality of a node is selected as actors' clustering indicators in social networks.

Degree centrality can be divided into two evaluation dimensions. OutDegree centrality is defined as the dimension of the "power of acquiring WOM information" and InDegree centrality is defined as the dimension of the "power of releasing WOM information". InDegree and OutDegree are two indexes respectively. Degree centrality reflects the power of the actors in releasing information or acquiring information. Accordingly, if the actor has high InDegree centrality, he can be "concerned" by more other nodes. Then the release and comments of WOM information can be noticed and known immediately, which means he has the greater "power" in releasing information. 
Betweenness centrality reflects the "power" of controlling the information flow. So a user's high degree of Betweenness centrality means his role of "bridge contacts" among many users. That is to say, if other users have the high dependency on the above mentioned users to obtain information, the above mentioned users have the greater power in the network and can control the information flow to large extent. The more such positions an actor can occupy in the network, the higher degree of betweenness centrality he or she has, and there are more actors who can not contact each other without this actor (Luo Jiade, 2005). Therefore his or her "power" of controlling the information communication among other users is defined as the "power of controlling WOM dissemination channels" as an index.

Closeness centrality is used to measure an actor's independency. In contrast to the above mentioned centrality, the smaller the value of closeness centrality of the node is, the closer the node is to the hub of networks. The closer distance of the node from others means the less control from other nodes in obtaining information. Closeness centrality reflects simplicity of "power" which means the user makes the information quickly arrive at each user. In this process, this user communicates information with less dependence on other users. Therefore, the index is defined as "WOM dissemination independence" dimension, and here "inFarness" is chosen from UCNET output for the index.

Through observing this set of microblog users, all kinds of centrality are measured to study the clustering of actors in the process of IWOM information dissemination.

Clustering data of actors' power dimension in WOM are shown in Table 1:

Table 1. Clustering data of actors' power dimension in WOM

\begin{tabular}{ccccc}
$\begin{array}{c}\text { Power } \\
\text { Dimension }\end{array}$ & $\begin{array}{l}\text { obtaining } \\
\text { Dimension }\end{array}$ & $\begin{array}{l}\text { Releasing } \\
\text { Dimension }\end{array}$ & $\begin{array}{l}\text { Controlling } \\
\text { Dimension }\end{array}$ & $\begin{array}{c}\text { Independence } \\
\text { Dimension }\end{array}$ \\
\cline { 2 - 5 } user & OutDegree & InDegree & Betweenness & inFarness \\
\hline User 1 & 1 & 2 & 0 & 32 \\
\hline User 2 & 1 & 2 & 0 & 28 \\
\hline User 3 & 1 & 3 & 0 & 28 \\
\hline User 4 & 1 & 4 & 0.622 & 25 \\
\hline User 5 & 11 & 8 & 41.011 & 19 \\
\hline User 6 & 11 & 3 & 5.789 & 26 \\
\hline User 7 & 6 & 6 & 19.067 & 21 \\
\hline User 8 & 12 & 5 & 23.9 & 22 \\
\hline User 9 & 6 & 6 & 15.167 & 22 \\
\hline User 10 & 3 & 6 & 1.289 & 21 \\
\hline User 11 & 1 & 5 & 0 & 32 \\
\hline User 12 & 4 & 9 & 24.078 & 17 \\
\hline User 13 & 3 & 3 & 1 & 25 \\
\hline User 14 & 7 & 6 & 16.578 & 21 \\
\hline & & & & \\
\hline
\end{tabular}

Stratified Cluster Analysis (Hierarchical Cluster order) is one of the most widely used methods in the Cluster Analysis. The clustering process is described as a merging of clusters based on their proximity. Each sample (or variable) is regarded as a group and then two or more groups belong to the same cluster if they are "close" according to a given distance or proximity until all the samples (or variables) can belong to a bigger cluster.

Clustering analysis of data in table1 is made by use of SPSS 17.0. Between groups linkage is employed here as the clustering method. 
Hierarchical Cluster tree is as follows:

Rescaled Distance Cluster Combine

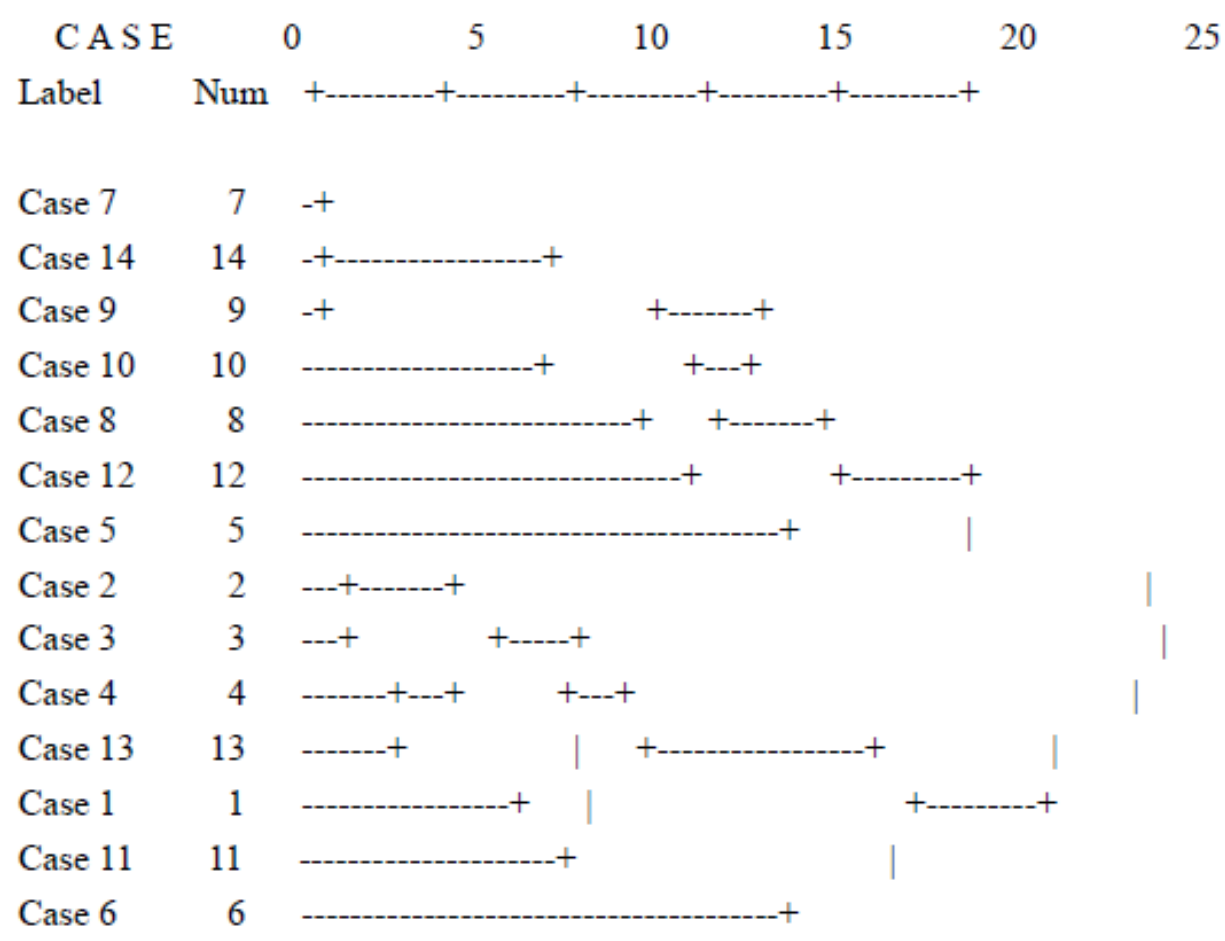

Figure 1. Stratified cluster tree

In Figure 1, clustering results show nodes can be divided into three categories. Among all the users, users $5,7,8,9$, $10,12,14$ show higher value in the power of controlling WOM dissemination channels; Users 1, 2, 3, 4, 11, 13 show the lower power of acquiring WOM information and lower power of releasing WOM information and have the lower power of controlling WOM dissemination channels. The user 6 belongs to an independent category with the lower releasing power of WOM information and the lower power of controlling WOM dissemination channels but the higher power of acquiring WOM information.

\section{Discussion and Implications}

1). The centricity can reflect actors' status in the WOM dissemination networks. "Power" is one of the sociological concepts. From the perspective of social network, a person has the power because he or she is related to others and can affect others. Network structures in social networks determines the members" "power" in networks and the "power" in WOM dissemination can be understood as factually existing or potential interaction models between WOM information publishers and recipients. The "power" affects the WOM information dissemination. Here "power" refers to word-of-mouth dissemination process between the actors in a reflects the interaction modes attributes rather than the members' own attributes in social networks. Social networks define power quantitatively from the perspective of "relationship", and give the specific and formalized definition of social power. That is to say, different kinds of centrality indexes are used to describe power.

2). IWOM information is characterized by low transmission cost, clearer targets and large range of transmission, effectively overcoming inherent defects of traditional WOM dissemination such us low efficiency and the small range. Henning-Thurau etc. think IWOM is the positive and negative comments on the company and its products communicated by potential, actual or former customers through the internet media (Hennig-Thurau, Thorsten, Kevin P. Gwinner, Gianfranco Walsh \& Dwayne D. Gremler, 2004).

Different from advertisement, IWOM is informal and noncommercial, so it will be the important way to obtain information (Zhang Xiaofei \& Dong Dahai, 2008). The transition from traditional WOM to IWOM is reflected in the centrality of social networks. Essentially, more actors' "WOM acquiring power" and "WOM releasing power" increase and the centrality of the diagram makes obvious changes accordingly. 
Due to the decrease in WOM dissemination cost, betweenness centrality shown in the social networks of IWOM is obviously much lower than that in the social networks of traditional WOM. And usually actors' power of controlling WOM dissemination channels gets decreasing. That is, in the IWOM communication, most actors in the whole social network can get WOM information without needing other nodes as bridge contact points. Personal information release platform in Web2.0 environment has the control of strong discourse power. So as to decentralizing the discourse power, it is very hard for marketers to control the communication channels of all the messages from up to down. Therefore, it is time that marketing managers transformed his status from "controllers" of information transmission to "participants" of information communication.

3). The relationship intensity is always an important variable in the existing literature on the WOM effect and its influence on consumers' decision-making. According to relationship intensity between senders and recipients, WOM can be divided into weak-tied WOM communication and strong-tied WOM communication. The intensity of the connections between the actors isn't involved in this paper, the study only analyzes the "concerned" and "concerning" relationship existing between actors in WOM information. Bansal \& Voyer (2000) studied WOM influence on consumer decision-making, and indicated that cases under the strong ties have stronger influence than weak ties cases (Bansal H S \& Voyer P A, 2000). Xi He etc (2008) pointed out that relationship intensity between actors in IWOM is weak ties, the trust tendency in IWOM exerts greater influence on WOM effect than in traditional WOM (Xi He, Xu Jinfa, Luo Shixin \& Huang Guoqun, 2008). Researchers attempt to define strong ties and weak ties in WOM from different angles, such as from the familiarity between senders and recipients of WOM information or from the strong or weak relationship in social networks suggested by Granovetter (1973).

In this paper, the relationship of concern existing in the "concern matrix" can not be distinguished easily by strong or weak relationship. The relationship intensity of concern need more specific empirical analysis. Undeniably, compared to the information source which actors in social networks fail to concern consistently and fixedly, the information source concerned consistently for a long time from which actors are more likely to acquire WOM information is the base of establishing the long-term stable trust.

The analysis of WOM concern tendency and information transmission path from the perspective of social network can show that WOM information transmission tend to be influenced by actors' "power".

Through centrality clustering of the social network mentioned above, WOM marketing managers can classify actors' "power" of WOM communication and make more targeted communication to find potential opinion leaders, strengthen marketing persuasive effect according to different power dimensions and actors' position in the network.

4). Social network can be used to make a static analysis of actors' power in WOM communication at a certain time. From the perspective of social networks of WOM communication, WOM communication network is a dynamic system, whose structure will change constantly within the duration (Luo Xiaoguang \& Yu Hongyi, 2009). In the real social network of WOM communication, the "concern" network between actors may be a changing dynamic network rather than a stable one formed in microblogs and other virtual social networks. Brown \& Broderick (2007) put forward the concept of Consumers-Website Relationship, and a web site is regarded as one actor in the online social network (Brown J, Broderick A J \& Lee N., 2007).

In the directed graph of WOM communication social network, behavioral centrality analysis of WOM information communication not only includes WOM communicating individuals but also information release platform concerned by the majority of people. That is, most of consumers choose some information release platforms through one-way "concern" relationship and then WOM information released in the information release platforms with "half broadcasting" mechanism can be found purposely or purposelessly in the process of consumers' search for information.

These highly concerned information platforms lie in the core position of "bridge". Their advantage in core position determines their advantage in information because the WOM information release from the position of "bridge" has the significant influence on group attitude in the internal network.

\section{Conclusions}

This article attempts to study WOM communication from the perspective of social networks. However, due to the small sample here, it can not describe a large-scale network structure. It is hoped that this paper can throw a new light on the WOM communication study by analyzing actors' different "power" dimensions.

In the future, if longitudinal and dynamic analysis of actors" "power" in WOM communication based on time series analysis can be made and information platforms can be included in actors of WOM communication social network, then more valuable conclusions will be made. 


\section{References}

Bansal, H. S., \& Voyer, P. A. (2000). Word of Mouth Processes within a Services Purchase Decision Context. Journal of Service Research, 3(2), 166-177.

Bristor, J. M. (1990). Enhanced explanations of word-of-mouth communications: The power of relationships. Research in consumer behavior, 4, 51-83.

Brown, J., \& Reingen, P H. (1987). Social ties and word-of-mouth referral behavior. Journal of Consumer Research, 14(12), 350-362.

Brown, J., Broderick, A. J., \&Lee, N. (2007). Word of mouth communication within online communities: Conceptualizing the online social network. Journal of Interactive Marketing, 21(3), 2-20.

Freeman, L. C. (1977). A Set of Measures of Centrality Based on Betweenness. Sociometry, 40, 35-41.

Freeman, L. C. (1979). Centrality in social networks: Conceptual clarification. Social Networks, 1, $215-239$.

Hennig-Thurau, Thorsten, Kevin, P. Gwinner, Gianfranco, Walsh, \& Dwayne, D. Gremler. (2004). Electronic Word-of-Mouth via Consumer-Opinion Platforms: What Motivates Consumers to Articulate Themselves on the Internet?. Journal of Interactive Marketing, 18(1), 38-52.

Li, Lin, Sun, Jun-Hua, \& Zhou, Zhang-Jin. (2009). The dissemination of service quality word-of-mouth based on complex social network. System Engineering, 2(6), 1-7.

Luo, Jiade. (2005). Social network analysis. Beijing, Social Sciences Academic Press, 96-98.

Luo, Xiaoguang, \& Yu, Hongyin. (2009). Formation and development of Customer Word of mouth Dissemination network. Science and management, 11(2),119-122.

Tang, Jianmin, \& Zhu, Sai. (2008). Study of word-of-mouth dissemination based on on social network analysis method. Spine Collective Economy, Z1, 85-86.

$\mathrm{Xi}$, He, Xu, Jinfa, Luo, Shixin, \& Huang, Guoqun. (2008). Comparative study between traditional WOM and online WOM effects on consumers' purchasing decisions. Finance \&Trade Economics, 2, 98-104.

Yang, Xue-Cheng, \& Zhang, Xiao-Hang. (2009). Application of Social network analysis in marketing. Contemporary Economic Management, 31(6), 25- 29.

Yu, Hongyin. (2009). Research on Word-of-mouth Dissemination Network between Customers. Harbin Institute of Technology University.

Zhang, Xiaofei, \& Dong, Dahai. (2008). Online WOM strategy. Marketing Guide, 6, 61-64. 